\title{
PARÁMETROS SOCIOLINGÜÍSTICOS EN LA ELECCIÓN DE LA PRIMERA PERSONA EN HIPÓLITO DE EURÍPIDES
}

\author{
MARÍA PEREIRA Rico \\ Universidad Autónoma de Madrid
}

\section{RESUMEN}

Este artículo se refiere a la influencia de los parámetros sociolingüísticos de edad, jerarquía social y género en la elección de la primera persona del singular y del plural por parte de los personajes de la tragedia de Eurípides Hipólito. Los datos extraídos de dicha tragedia mostrarán que existe una distribución del singular y del plural en función del poder que posean los personajes, de tal manera que aquellos con mayor importancia social acudirán al singular en mayor medida, mientras que el plural se encontrará con mayor frecuencia en boca de los personajes inferiores en jerarquía social. Asimismo, el estudio de los contextos mostrará en qué circunstancias utilizan aquellos el plural y en cuáles los personajes no libres acuden al singular. Por tanto, se deduce que la influencia principal viene ejercida por el parámetro de jerarquía social, mientras que los de género y edad se encuentran en un segundo plano.

Palabras clave: contexto, poder, clase social, parámetros sociolingüísticos, identidad.

\section{ABSTRACT}

This paper deals with the influence of the sociolinguistic patterns of age, social class, and gender on the choice of the first person singular and plural by the characters of Euripides' tragedy Hippolytus. The data taken out from the mentioned tragedy will show that there is a relation between the choice of the singular vs. plural first person and the social position of the speaker, so that those characters with more social importance will use the singular more frequently, whereas the plural will be found used more often by the characters of a lower social position. Likewise, the study of the contexts will indicate the circumstances in which the former use the plural and those in which the non-free characters employ the singular. Thus, the main influence is exerted by the pattern of social class, whilest gender and age can be considered secundary influences in this respect.

Key Words: context, power, social class, sociolinguistic patterns, identity.

\section{INTRODUCCIÓN}

A lo largo de este trabajo analizaré y desarrollaré desde una perspectiva sociolingüística algunas de las conclusiones que se desprenden 
del estudio del empleo de las formas verbales en la primera persona del singular y del plural por parte de los personajes que participan en Hipólito de Eurípides ${ }^{1}$.

El motivo que me lleva a abordar dicho tema es el hecho de que el uso de dichas personas verbales puede arrojar información relevante sobre la posición social que ostentan los personajes que hacen uso de ellas, prestando especial atención a los parámetros sociolingüísticos de edad, jerarquía social y género ${ }^{2}$.

Además, el estudio de estas formas verbales es importante por su contenido deíctico. El hecho de que el hablante se señale a sí mismo como sujeto da lugar a una mayor subjetividad en el discurso, más personal, con mayor marcación de la identidad, especialmente, con relación a los personajes libres, como se verá.

Por otra parte, es de crucial importancia destacar que el uso de estas personas verbales debe ser tratado en su contexto y no de manera aislada, es decir, no solo es preciso observar qué personajes acuden a la primera persona del singular o del plural en mayor medida, sino que también es necesario considerar en qué situaciones y frente a quién se hace uso de ellas. Partiendo de esta base, es importante destacar que el lenguaje debe ser tratado siempre dentro de un contexto, de tal manera que será lógico pensar que los personajes puedan variar su registro y estilo en relación con su interlocutor, así como con la situación conversacional en la que se encuentren.

Teniendo en cuenta esta idea, se podrá entender que el empleo de la primera persona no tiene los mismos efectos o connotaciones en todas las situaciones conversacionales; lo que en algunos contextos puede considerarse ostentación de poder, en otros, atendiendo también a la semántica, puede reflejar algún otro tipo de comportamiento.

1 El desarrollo de distintos trabajos en los que se presta especial atención a los parámetros sociolingüísticos, fundamentalmente en lo que se refiere al género, se ha introducido también en los estudios clásicos. Así, destacan los elaborados por Calame 1995 o McClure 1995, 1999, aunque estos se centran en el punto de vista literario más que lingüístico.

${ }^{2}$ Se puede observar cierta conexión entre el estudio que aquí se presenta y los trabajos que se dedican al análisis del uso de la primera persona desde una perspectiva narratológica. En este sentido, destacan las observaciones realizadas por Minchin 2007 respecto a los protagonistas de las historias contadas en los poemas homéricos o las llevadas a cabo por Berman 1998, p. 66: «[...]The Javanese stories we examine, however, fall into several types, or genres, of which only those told by the high-ranking or elderly speakers show similarities to the structure of narrative preciously mentioned. Participants who are not high-ranking, either through age or through achievement $[\ldots]$, or within the bounded context of interaction, that is, in comparison to their immediate speech partners, do not have the right to tell autobiographical stories. While the conversational narrative as a bounded speech event is always interactive, the differences here lie in cultural variations concerning the individual in society». 


\section{Metodología}

La metodología utilizada para la extracción de los datos responde a un recuento manual de todos los verbos que se han encontrado en las formas de primera persona del singular y del plural y su posterior clasificación atendiendo a los personajes que acuden a dichas formas.

En segundo lugar, se ha confeccionado una serie de tablas para poder exponer con mayor claridad los datos extraídos. En cada una de estas tablas, se pueden observar los usos de la primera persona del singular y del plural por parte de los distintos personajes que participan en Hipólito.

Así, cada una de ellas refleja de forma independiente la utilización de estas formas por cada uno de los personajes en dos tipos de contextos, en aquellos en los que existe diálogo y en los que el discurso se encuentra en monólogo ${ }^{3}$, mostrando el número de veces que el personaje en cuestión emplea dichas personas verbales, el número de versos en los que se encuentran los ejemplos -en nota a pie de página-, y el porcentaje que refleja la cantidad de veces que ese personaje acude a dichas personas verbales tomando como base el número total de ejemplos de formas en primera persona del singular y del plural que aparecen en todos los personajes.

Por último, creo conveniente indicar que la edición que se ofrece en los ejemplos de este estudio es la de Diggle 1984, publicada por Oxford University Press, y la traducción al castellano es la realizada por J. A. López Férez y J. M. Labiano (Crespo 2004). Además, cabe destacar que aparecen en cursiva los términos que se pretenden resaltar.

\section{EXPOSICIÓN DE LOS DATOS}

En las tablas 1-9, se puede observar cómo se refleja el empleo de las primeras personas verbales -en singular y en plural- por parte de cada uno de los personajes de Hipólito.

\footnotetext{
${ }^{3}$ La importancia del contexto viene dada por la variación que la intención pragmática y semántica puede sufrir dependiendo de la situación conversacional en la que se integre. La división entre contextos de diálogo y contextos de monólogo, o más bien, de relato y no-relato se ha utilizado en los últimos tiempos especialmente en los estudios de marcadores discursivos. Una de las primeras alusiones en este sentido aparece en Schiffrin 1987, p. 14, quien defiende las diferencias de expresión que pueden ofrecerse dependiendo del contexto en el que se integren tales marcadores. También es importante la descripción de narratividad expuesta por Greimas y Courtés 1990, p. 272. Siguiendo esta línea, he considerado relevante introducirlos también en este estudio, puesto que la implicación que se desprende varía notablemente de un tipo de contexto a otro. Para el estudio de las relaciones de poder entre los distintos personajes basándonos en el uso de la primera persona, por tanto, será mucho más interesante observar el comportamiento de tales personajes en contextos de diálogo del que exista en monólogo.
} 
Asimismo, es conveniente llamar la atención sobre el hecho de que en dichas tablas solo se han tenido en cuenta aquellas formas verbales en primera persona, a pesar de que en algún contexto se pueda hacer referencia a esta primera persona a través de algún elemento diferente al de la utilización de la desinencia verbal. Me estoy refiriendo a los verbos impersonales o formas verbales no personales que apuntan hacia la primera persona a través de otro tipo de procedimientos.

(1) $\varphi \varepsilon \tilde{v}$.

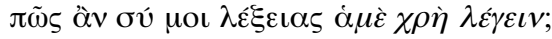

¿Cómo podrías decirme tú lo que he de decir?

(Hip. v. 345)

Estos casos no han sido tratados en el posterior análisis puesto que, aunque la introducción del pronombre lleva a una mayor marcación, su alternancia con el plural resulta muy escasa, puesto que no aparece en ningún caso como sujeto de una oración de infinitivo ${ }^{4}$. Este hecho, por tanto, conduce a una falta de datos -al menos, en la obra que sirve como objeto de análisis- a la hora de poder observar la alternancia de uso entre el singular y el plural por parte de los distintos personajes, finalidad primera de este trabajo.

Por otro lado, cabe destacar la diferencia existente en griego entre el plural inclusivo y el plural exclusivo ${ }^{5}$. Esta diferencia se hace imprescindible por las variaciones de significado e implicación que puede aportar

${ }^{4}$ Solo aparece como objeto directo también con este significado de plural de modestia. En el ejemplo de Fedra que se muestra a continuación y en dos ocasiones en sendas inter-

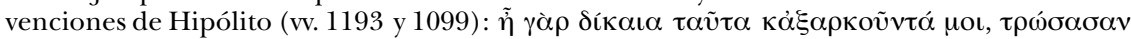

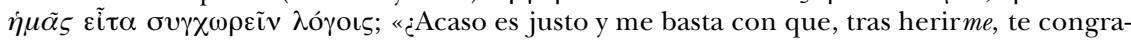
cies de palabra conmigo?» (Hip. vv. 702-703).

${ }_{5}^{5}$ Terminología utilizada por Benveniste 2004, pp. 168 y ss. para aludir a dos conceptos distintos de plural que se formulan a partir de la primera persona del plural, cuya diferencia radica en las personas que se encuentran encuadradas dentro del nosotros. El inclusivo implicaría la actuación del yo + tú / vosotros, mientras que el exclusivo indicaría la manifestación del yo + ellos. En palabras del propio Benveniste: «Esta denominación de inclusivo y exclusivo no puede tenerse por satisfactoria; descansa de hecho en la inclusión o la exclusión del vosotros, mas por relación a ellos, las designaciones podrían invertirse exactamente. Sin embargo, será difícil hallar términos más apropiados. De mayor importancia nos parece el análisis de esta categoría inclusivo-exclusivo desde el punto de vista de las relaciones de persona. Aquí el hecho esencial que hay que reconocer es que la distinción de las formas inclusiva y exclusiva se moldea en realidad sobre la relación que planteamos entre la $1^{\underline{a}}$ y la $2^{\underline{a}} \operatorname{sg}$., y entre la $1^{\underline{a}}$ y la $3^{\underline{a}} \operatorname{sg}$., respectivamente». En este sentido, se pueden encontrar otros trabajos en los que se compara este uso del plural inclusivo y exclusivo en distintas lenguas, entre los que destaca el realizado por Cysouw 2003, que se dedica al análisis interlingüístico de estas formas con una mención especial a lenguas en las que existen diferencias morfológicas en la formación de esta clase de plural. Además, al igual que hace Lyons 1968, p. 277, se destaca la dificultad de utilizar el término «plural» para las personas verbales, puesto que no responden a un grupo de personas que realiza una acción de forma plural, a la vez, sino más bien a la intención de estos de incluirse dentro de un grupo en el que normalmente uno de ellos realiza esa acción de forma principal. 
a la obra y a la actitud de los distintos personajes. Cabe pensar que los personajes con una categoría social inferior a aquel con el que están dialogando utilizarán con mayor frecuencia el plural exclusivo -puesto que su estatus inferior no les permite incluirse en una misma persona verbal con su interlocutor, de estatus superior-, mientras que los personajes con mayor importancia social acudirán al inclusivo. Los datos son muy escasos para confirmar esta idea, sin embargo, sí parece que esta tendencia se cumple en los personajes de condición más humilde. Ahora bien, la Nodriza, como en otros casos, representa la excepción respecto a esta tesis, ya que utiliza el plural inclusivo con Fedra en un alto porcentaje. Será, en este caso, su papel funcional el que explicará tal uso.

Además, es importante señalar en este punto la utilización del llamado plural de modestia -del que hablaré más adelante- porque, aunque en principio se trata de un plural inclusivo, su referente no es otro que el hablante mismo en singular ${ }^{6}$.

En los datos que se muestran a continuación, se ha añadido una fila independiente dentro de la primera persona del plural en aquellos personajes que hacen uso del llamado plural de modestia, así como se han señalado en cursiva dentro del plural -en notas a pie de página- aquellos ejemplos que, dentro de este empleo, no se refieren exclusivamente al hablante y receptor o receptores, sino que aluden a un grupo más numeroso de miembros, entre los que se cuenta el personaje, como el conjunto de todos los mortales o de todas las mujeres en alguno de los ejemplos pronunciados por los personajes femeninos, como la Nodriza; serían estos datos correspondientes al plural exclusivo.

Por último, es preciso hacer notar que en las tablas se expresa de manera explícita la diferencia entre la primera persona del plural y la primera persona del singular utilizadas por cada uno de los personajes cuando estos lo hacen dentro de un discurso con forma de monólogo y cuando lo hacen en un discurso de interacción con otro personaje en diálogo, como ya se ha expuesto. La diferencia de uso de estos en lo que se refiere a la expresión de poder, por tanto, solo podrá estudiarse en los momentos de interacción, puesto que en el resto de los contextos no será relevante para el fin que aquí se está intentando llevar a cabo ${ }^{7}$.

\footnotetext{
${ }^{6}$ El plural de modestia se utiliza por parte de un interlocutor cuyo referente es solo él mismo, pero que, a diferencia del mayestático, al que se acude cuando el hablante pretende mostrar mayor poder frente a su interlocutor o interlocutores, se utiliza como estrategia para evitar cierta responsabilidad ante el hecho que se describe. Este tipo de plural, junto con el mayestático y el asociativo, aparecen citados de forma tangencial en algunas gramáticas griegas, como es el caso de Lasso de la Vega 1968, p. 293 o con anterioridad la de Gildersleeve 1900, p. 27, en la que ya se observa que el uso de la primera persona del plural en lugar del singular se debe a factores de modestia.
}

7 He considerado contextos de diálogo también los momentos de invocación hacia un dios. 
TABLa 1.

\begin{tabular}{|c|c|c|c|c|}
\hline \multirow[b]{2}{*}{ AFRODITA } & \multirow{2}{*}{$\begin{array}{l}\text { Número } \\
\text { de veces } \\
\text { que se } \\
\text { presenta }\end{array}$} & \multicolumn{2}{|c|}{$\begin{array}{l}\text { Porcentaje respecto } \\
\text { a toda su intervención }\end{array}$} & \multirow{2}{*}{$\begin{array}{l}\text { Porcentaje } \\
\text { respecto } \\
\text { a la expresión } \\
\text { aquí tratada }\end{array}$} \\
\hline & & Monólogo & Diálogo & \\
\hline $\begin{array}{l}1^{\text {a }} \text { persona } \\
\text { del singular }\end{array}$ & $10^{8}$ & $10 / 36=27.8 \%$ & & $10 / 311=3.2 \%$ \\
\hline \multicolumn{5}{|l|}{$\begin{array}{l}1^{\underline{a}} \text { persona } \\
\text { del plural }\end{array}$} \\
\hline Total & 10 & $10 / 36=27.8 \%$ & & $10 / 311=3.2 \%$ \\
\hline
\end{tabular}

TABLA 2.

\begin{tabular}{|c|c|c|c|c|}
\hline \multirow[b]{2}{*}{ HIPÓLITO } & \multirow{2}{*}{$\begin{array}{l}\text { Número } \\
\text { de veces } \\
\text { que se } \\
\text { presenta }\end{array}$} & \multicolumn{2}{|c|}{$\begin{array}{l}\text { Porcentaje respecto } \\
\text { a toda su intervención }\end{array}$} & \multirow{2}{*}{$\begin{array}{c}\text { Porcentaje } \\
\text { respecto } \\
\text { a la expresión } \\
\text { aquí tratada }\end{array}$} \\
\hline & & Monólogo & Diálogo & \\
\hline $\begin{array}{l}1^{\underline{a}} \text { persona } \\
\text { del singular }\end{array}$ & $85^{9}$ & $49 / 238=20.6 \%$ & $36 / 238=15.1 \%$ & $85 / 311=27.3 \%$ \\
\hline $\begin{array}{l}1^{\underline{a}} \text { persona } \\
\text { del plural }\end{array}$ & $4^{10}$ & & $4 / 238=1.7 \%$ & \multirow{2}{*}{$9 / 311=2.9 \%$} \\
\hline $\begin{array}{l}\text { plural } \\
\text { de modestia }\end{array}$ & $5^{11}$ & & $5 / 238=2.1 \%$ & \\
\hline Total & 94 & $94 / 238=39.5 \%$ & & $94 / 311=30.2 \%$ \\
\hline
\end{tabular}

${ }^{8}$ VERSOS: 2, 5, 6, 9, 20, 21, 42, 48, 51, 53.

${ }^{9}$ VERSOS EN MONÓLOGO: 74, 85(*2), 87(*2), 640, 653, 654, 655, 657, 658, 660, 661, 663, 664, 902, 996, 1004, 1006, 1008, 1011, 1012, 1017, 1022, 1023, 1026, 1028, 1031, 1033, 1060, 1061(*2), 1062, 1063(*2), 1066, 1067, 1091(*3), 1097, 1349, 1350, 1354, 1366, 1369, 1375, 1385(*2). VERSOS EN DIÁLOGO: 92, 102, 112, 113, 602, 604, 614, 904(*2), 905, 907, 910, 934, 991, 1041, 1043(*2), 1071(*2), 1075, 1078, 1079, 1392(*2), 1395, 1401, 1403, 1405, 1409, 1442, $1443,1447(* 2), 1449,1451,1457$.

10 VERSOS: $60,90,98,626$.

11 VERSOS: 660, 933, 1035, 1079, 1093. 
TABLA 3.

\begin{tabular}{|l|c|c|c|c|}
\hline \multirow{2}{*}{$\begin{array}{c}\text { SIRVIENTE } \\
\text { Número }\end{array}$} & $\begin{array}{c}\text { Ne veces } \\
\text { que se } \\
\text { presenta }\end{array}$ & \multicolumn{2}{|c|}{$\begin{array}{c}\text { Porcentaje respecto } \\
\text { a toda su intervención }\end{array}$} & $\begin{array}{c}\text { Poncentaje } \\
\text { Pólogo } \\
\text { respecto } \\
\text { a la expresión } \\
\text { aquí tratada }\end{array}$ \\
\hline $\begin{array}{l}1^{\underline{a}} \text { persona } \\
\text { del singular }\end{array}$ & $3^{13}$ & $3 / 25=12 \%$ & Diálogo & $3 / 311=1 \%$ \\
\hline $\begin{array}{l}1^{\underline{a}} \text { persona } \\
\text { del plural }\end{array}$ & $3^{14}$ & $2 / 25=8 \%$ & $1 / 25=4 \%$ & $3 / 311=1 \%$ \\
\hline Total & 6 & $6 / 25=24 \%$ & $6 / 311=1.9 \%$ \\
\hline
\end{tabular}

TABLA 4.

\begin{tabular}{|l|c|c|c|c|}
\hline \multirow{2}{*}{ CORO } & \multirow{2}{*}{$\begin{array}{c}\text { Número } \\
\text { de veces } \\
\text { que se } \\
\text { presenta }\end{array}$} & \multicolumn{2}{|c|}{$\begin{array}{c}\text { Porcentaje respecto } \\
\text { a toda su intervención }\end{array}$} & $\begin{array}{c}\text { Porcentaje } \\
\text { respecto } \\
\text { a la expresión } \\
\text { aquí tratada }\end{array}$ \\
\cline { 3 - 5 } $\begin{array}{l}1^{\text {a }} \text { persona } \\
\text { del singular }\end{array}$ & $22^{15}$ & $9 / 158=5.7 \%$ & $13 / 158=8.2 \%$ & $22 / 311=7.1 \%$ \\
\hline $\begin{array}{l}1^{\underline{a}} \text { persona } \\
\text { del plural }\end{array}$ & $5^{16}$ & $3 / 158=1.9 \%$ & $2 / 158=1.3 \%$ & $5 / 311=1.6 \%$ \\
\hline Total & 27 & $27 / 158=17.1 \%$ & $27 / 311=8.7 \%$ \\
\hline
\end{tabular}

${ }^{12}$ Los versos que se corresponden con el pasaje que abarca del 1100 al 1120 son de atribución dudosa, la lectura que aquí se ofrece los incluye en el parlamento del Sirviente, pero se puede suponer también que pertenecen al Coro.

13 VERSOS: $1106,1122(* 2)$.

14 VERSOS EN MONÓLOGO: 1124(*2). VERSOS EN DIÁLOGO: 116.

15 VERSOS EN MONÓLOGO: 135, 364, 732, 735, 743, 1119, 1143, 1146, 1151. VERSOS EN DIÁLOGO: $483,568,585(* 2), 592,713,788,805,855,869,873,981(* 2)$.

16 VERSOS EN MONÓLOGO: 268, 270, 540. VERSOS EN DIÁLOGO: 782, 804. 
TABLA 5.

\begin{tabular}{|c|c|c|c|c|}
\hline \multirow[b]{2}{*}{ MENSAJERO } & \multirow{2}{*}{$\begin{array}{l}\text { Número } \\
\text { de veces } \\
\text { que se } \\
\text { presenta }\end{array}$} & \multicolumn{2}{|c|}{$\begin{array}{l}\text { Porcentaje respecto } \\
\text { a toda su intervención }\end{array}$} & \multirow{2}{*}{$\begin{array}{l}\text { Porcentaje } \\
\text { respecto } \\
\text { a la expresión } \\
\text { aquí tratada }\end{array}$} \\
\hline & & Monólogo & Diálogo & \\
\hline $\begin{array}{l}1^{\underline{a}} \text { persona } \\
\text { del singular }\end{array}$ & $9^{17}$ & $5 / 70=7.1 \%$ & $4 / 70=5.7 \%$ & $9 / 311=2.9 \%$ \\
\hline $\begin{array}{l}1^{\mathrm{a}} \text { persona } \\
\text { del plural }\end{array}$ & $6^{18}$ & $6 / 70=8.6 \%$ & & $6 / 311=1.9 \%$ \\
\hline Total & 15 & $15 / 70=21.4 \%$ & & $15 / 311=4.8 \%$ \\
\hline
\end{tabular}

TABLA 6.

\begin{tabular}{|l|c|c|c|c|}
\hline \multirow{2}{*}{ NODRIZA } & \multirow{2}{*}{$\begin{array}{c}\text { Número } \\
\text { de veces } \\
\text { que se } \\
\text { presenta }\end{array}$} & \multicolumn{2}{|c|}{$\begin{array}{c}\text { Porcentaje respecto } \\
\text { a toda su intervención }\end{array}$} & $\begin{array}{c}\text { Porcentaje } \\
\text { respecto } \\
\text { a la expresión } \\
\text { aquí tratada }\end{array}$ \\
\cline { 3 - 5 } $\begin{array}{l}1^{\text {a }} \text { persona } \\
\text { del singular }\end{array}$ & $39^{19}$ & $6 / 209=2.9 \%$ & $33 / 209=15.8 \%$ & $39 / 311=12.5 \%$ \\
\hline $\begin{array}{l}1^{\underline{a}} \text { persona } \\
\text { del plural }\end{array}$ & $10^{20}$ & & $10 / 209=4.8 \%$ & $10 / 311=3.2 \%$ \\
\hline Total & 49 & $49 / 209=23.4 \%$ & & $49 / 311=15.7 \%$ \\
\hline
\end{tabular}

${ }^{17}$ VERSOS EN MONÓLOGO: 1182, 1191(*2), 1245, 1248. Los ejemplos que aparecen en el verso 1182 y los dos que se encuentran en el 1191 están en boca del Mensajero, pero corresponden a Hipólito, es decir, se trata de un pasaje en estilo directo: aquel reproduce las palabras que este ha pronunciado anteriormente. VERSOS EN DIÁLOGO: 1154, 1157, 1250, 1254.

18 VERSOS: 1174, 1187, 1196, 1198, 1206, 1244.

19 VERSOS EN MONÓLOGO: $177(* 2), 250,260,264,458$. VERSOS EN DIÁLOGO: 271, 284(*2), 285, 287, 291, 292, 298, 324, 326, 342, 344(*2), 346, 354, 355, 356(*3), 357, 435, 496, 517, 521, 523, 697, 698(*2), 699(*2), 700(*2), 704.

${ }^{20}$ VERSOS: 193, 197, 289, 301, 302, 450, 481, 701, 704, 781. 
TABLA 7.

\begin{tabular}{|l|c|c|c|c|}
\hline \multirow{2}{*}{ FEDRA } & \multirow{2}{*}{$\begin{array}{c}\text { Número } \\
\text { de veces } \\
\text { que se } \\
\text { presenta }\end{array}$} & \multicolumn{2}{|c|}{$\begin{array}{c}\text { Porcentaje respecto } \\
\text { a toda su intervención }\end{array}$} & $\begin{array}{c}\text { Porcentaje } \\
\text { Monólogecto } \\
\text { respecto } \\
\text { la expresión } \\
\text { aquí tratada }\end{array}$ \\
\cline { 3 - 4 } $\begin{array}{l}1^{\text {a }} \text { persona } \\
\text { del singular }\end{array}$ & $52^{21}$ & $26 / 167=15.6 \%$ & $26 / 167=15.6 \%$ & $52 / 311=16.7 \%$ \\
\hline $\begin{array}{l}1^{\text {a }} \text { persona } \\
\text { del plural }\end{array}$ & $4^{22}$ & $4 / 167=2.4 \%$ & & $11 / 311=3.5 \%$ \\
\hline $\begin{array}{l}\text { plural } \\
\text { de modestia }\end{array}$ & $7^{23}$ & & $7 / 167=4.2 \%$ & \\
\hline Total & 63 & $63 / 167=37.7 \%$ & $63 / 311=20.2 \%$ \\
\hline
\end{tabular}

TABLA 8.

\begin{tabular}{|l|c|c|c|c|}
\hline & \multirow{2}{*}{$\begin{array}{c}\text { Número } \\
\text { de veces } \\
\text { que se } \\
\text { ÁRTEMIS }\end{array}$} & \multicolumn{2}{|c|}{$\begin{array}{c}\text { Porcentaje respecto } \\
\text { a toda su intervención }\end{array}$} & $\begin{array}{c}\text { Porcentaje } \\
\text { prespecto } \\
\text { a la expresión } \\
\text { aquí tratada }\end{array}$ \\
\cline { 3 - 4 } $\begin{array}{l}1^{\text {a }} \text { persona } \\
\text { del singular }\end{array}$ & $11^{24}$ & Monólogo & Diálogo & $11 / 311=3.5 \%$ \\
\hline $\begin{array}{l}1^{\underline{a}} \text { persona } \\
\text { del plural }\end{array}$ & $1^{25}$ & $11 / 73=15.1 \%$ & $1 / 311=0.3 \%$ \\
\hline Total & 12 & $12 / 73=16.4 \%$ & $1 / 73=1.4 \%$ & $12 / 311=3.8 \%$ \\
\hline
\end{tabular}

${ }^{21}$ VERSOS EN MONÓLOGO: 199, 209, 211, 215, 219, 239, 240, 241(*2), 376, 388, 390, 391, 392, 393(*2), 399, 400, 405, 406, 413, 420, 421, 430, 673, 674. VERSOS EN DIÁLOGO: 230, 312, $315(* 2), 321,323,335(* 2), 341,504,506(* 2), 518,567,599,685(* 2), 686,709,716,719,720,723$, $727(* 2), 728$.

${ }^{22}$ VERSOS: $380\left(^{*} 2\right), 381,670$.

23 VERSOS: 244, 331, 349, 565, 575, 672, 688.

24 VERSOS: 1283, 1285, 1297(*2), 1298, 1332, 1396, 1422, 1425, 1435, 1439.

25 VERSO: 1341. 
TABLA 9.

\begin{tabular}{|c|c|c|c|c|}
\hline \multirow[b]{2}{*}{ TESEO } & \multirow{2}{*}{$\begin{array}{l}\text { Número } \\
\text { de veces } \\
\text { que se } \\
\text { presenta }\end{array}$} & \multicolumn{2}{|c|}{$\begin{array}{l}\text { Porcentaje respecto } \\
\text { a toda su intervención }\end{array}$} & \multirow{2}{*}{$\begin{array}{c}\text { Porcentaje } \\
\text { respecto } \\
\text { a la expresión } \\
\text { aquí tratada }\end{array}$} \\
\hline & & Monólogo & Diálogo & \\
\hline $\begin{array}{l}1^{\underline{a}} \text { persona } \\
\text { del singular }\end{array}$ & $33^{26}$ & $14 / 156=9 \%$ & $19 / 156=12.2 \%$ & $33 / 311=10.6 \%$ \\
\hline $\begin{array}{l}1^{\text {a }} \text { persona } \\
\text { del plural }\end{array}$ & $1^{27}$ & & $1 / 156=0.6 \%$ & \multirow{2}{*}{$2 / 311=0.6 \%$} \\
\hline $\begin{array}{l}\text { plural } \\
\text { de modestia }\end{array}$ & $1^{28}$ & & $1 / 156=0.6 \%$ & \\
\hline Total & 35 & \multicolumn{2}{|c|}{$35 / 156=22.4 \%$} & $35 / 311=11.2 \%$ \\
\hline
\end{tabular}

\section{ANÁlisis}

Como se puede observar, el personaje que más acude a la primera persona del singular es Hipólito, el protagonista, tanto en los discursos en forma de monólogo como en los diálogos. Este hecho no debería sorprender en absoluto, puesto que, como protagonista, constituye el centro del interés dramático.

Por otra parte, respecto al uso de la primera persona del plural, los datos no resultan tan claros al estudiarlos detenidamente. Aunque Fedra es la que parece acudir en mayor medida a esta forma, su utilización se reduce a la referencia a ella misma a través del plural o, en cualquier caso, a un empleo del plural en el que hace referencia al conjunto de las mujeres o de todos los mortales, plural exclusivo.

Además, cabe destacar en este punto que el análisis del uso de la primera persona en plural y en singular es mucho más interesante cuando, basando su estudio en la confrontación de ambas formas, se observa que la diferencia entre estas es menor, tal y como ocurre en el caso del Mensajero o del Sirviente. La ecuación, por tanto, sería la siguiente: a mayor diferencia entre singular y plural -inclinándose la balanza hacia el singular-, mayor poder presentará el personaje.

${ }^{26}$ VERSOS EN MONÓLOGO: 822, 827, 831, 836, 840, 845, 846, 956, 967, 971, 976, 1408, 1410, 1461. VERSOS EN DIÁLOGO: 806, 809, 817, 865, 877, 878, 879, 883, 893, 946, 950, 1054(*2), 1059, $1088,1260(* 2), 1267,1325$.

27 VERSO: 931.

28 VERSO: 1414. 
Así, el uso de estas personas verbales resulta especialmente relevante a la hora de analizar los datos desde una perspectiva sociolingüística, sobre todo en lo que se refiere al parámetro de jerarquía social y siempre que se manifiesten dentro de contextos de diálogo, de ahí la diferencia mostrada en las tablas.

La distribución que se encuentra en el empleo de estas formas por parte de los distintos personajes de la tragedia respondería al siguiente hecho: la situación social que viven los personajes libres les autoriza a utilizar en mayor medida el singular, mientras que el empleo de este no tiene tanta relevancia en los personajes no libres. De tal modo, la diferencia entre el porcentaje de uso del singular y del plural no se encuentra tan marcada como en aquellos.

De la misma forma, se puede observar que en los personajes libres, el parámetro de género parece condicionar la elección del plural. Los personajes femeninos acuden con mayor frecuencia a la primera persona del plural en comparación con el uso de esta forma que se encuentra en los masculinos. Esta situación reflejaría, a juzgar por los contextos, un comportamiento más autoritario por parte de estos últimos, frente a una mayor modestia y generalidad en los personajes femeninos.

La distribución de uso del singular en las partes en que existe diálogo en los personajes libres -especialmente en los personajes masculinos y divinidades ${ }^{29}$ - y el estudio desde el punto de vista semántico de los contextos en los que dicho empleo aparece invita a pensar que el singular estaría asociado al concepto de poder e identidad, y marcaría de manera más obvia su dominación hacia los personajes que no realizan un empleo tan frecuente de esta persona.

Así, por ejemplo, en la intervención de Hipólito el uso de la primera persona está asociado fundamentalmente con dos situaciones: por un lado, con la imposición y muestra de poder ante personajes de rango social inferior, como es el caso de (2), donde se encuentra dialogando con su sirviente; y por otro lado, con el lamento en primera persona y evidencias de impotencia ante las acusaciones de su padre en el agón que mantiene con él -ejemplo (3)-.

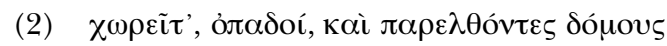

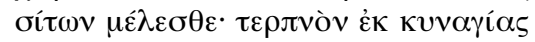

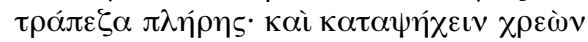

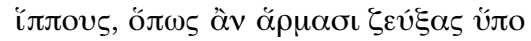

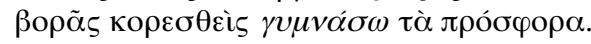

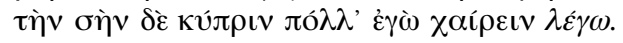

Marchad, compañeros, y, cuando entréis en la mansión, ocupaos de la comida. Cosa grata es una mesa llena después de la caza. Menester

\footnotetext{
${ }^{29}$ Habría que atender al uso de Ártemis, puesto que el de Afrodita solo se encuentra en monólogo.
} 
es almohazar los caballos, con el fin de que, tras uncirlos al carro y hartarme de comida, haga mis ejercicios convenientes. A tu Cipris le digo que lo pase bien.

(Hip. vv. 108-113)

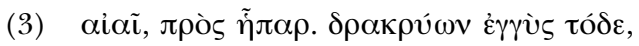

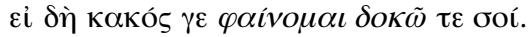

¡Ay, ay! Esto hiere mi corazón y me acerca a las lágrimas: que aparento ser malvado y te lo parezco a ti.

(Hip. vv. 1070-1071)

Por tanto, las diferencias en el uso de la primera persona del singular y del plural y las interpretaciones de tal empleo varían en función del significado global del contexto en el que estas formas aparecen.

En cuanto a los personajes libres, se podría decir que el uso de la primera persona del plural viene condicionado por tres circunstancias; en primer lugar, el empleo habitual de esta persona, en la que este incluye al emisor y al receptor -cuando se trata de un plural inclusivo, como en las palabras pronunciadas por Hipólito, ejemplo (4)-; en segundo lugar, aquellos versos en los que aparece mencionado también el conjunto de todos los mortales -plurales exclusivos como se aprecia en la intervención de Fedra, ejemplo (5)-; y por último, para hablar de sí mismos de manera más genérica.

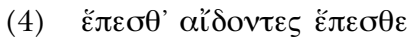

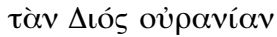

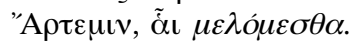

Seguidme, seguidme cantando a la celestial hija de Zeus, a Ártemis que se cuida de nosotros.

(Hip. vv. 58-60)

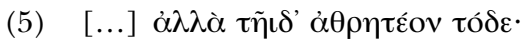

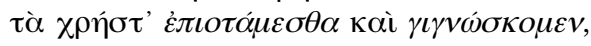

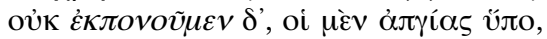

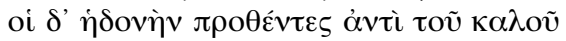

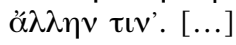

[...] Mas hay que examinarlo de la siguiente manera: sabemos y conocemos lo bueno, pero no lo realizamos; unos, por pereza; otros, porque prefieren cualquier otro placer en vez del bien.

(Hip. vv. 379-383)

Este último caso resulta bastante relevante según indican los datos, el llamado plural de modestia ${ }^{30}$, dado que deja entrever un menor grado

${ }^{30}$ Véase Gili y Gaya 1961, p. 229. 
de implicación por parte del hablante ante su afirmación. Generalmente, son los personajes libres los que hacen uso de esta estructura -Hipólito, Teseo y Fedra-, y acuden a él en aquellas situaciones en las que intentan evitar la responsabilidad que les correspondería ante la acción expresada. El caso más representativo es el de Fedra, cuyo uso de la primera persona del plural prácticamente se reduce a dicho plural de modestia -al menos, así sucede en los contextos de diálogo-.

A continuación, se muestran dos usos de la posible alternancia entre plural y singular de la que Fedra hace uso. En el primero de ellos, se observa la utilización del plural en su significado singular, en el que el propio traductor ha optado en castellano por el singular, frente al uso del plural del griego, mientras que en el segundo, se puede observar el uso del singular en un contexto semántico similar.

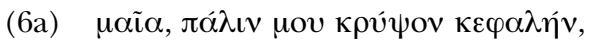

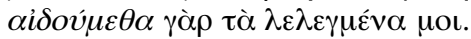

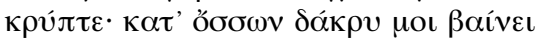

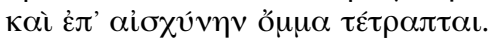

Madre, cúbreme de nuevo la cabeza, pues me ruborizo de lo que he dicho. Cúbreme. De mis ojos me llegan lágrimas, y mi rostro se ha vuelto de vergüenza.

(Hip. vv. 243-246)

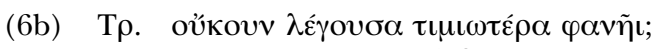

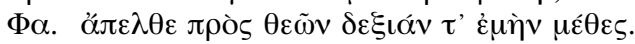

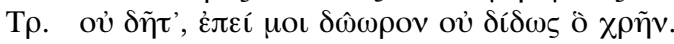



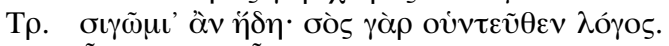

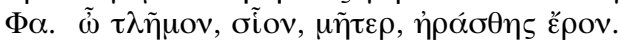

NoDriza: Pues si hablas, resultarás más honrada.

FEDRA: Apártate, por los dioses, y suelta mi mano derecha.

NoDriza: No, por supuesto, pues no me otorgas el don que debes.

FEDRA: Te lo otorgaré, pues siento vergüenza por respeto a tu mano. NODRIZA: Ya me callo, ya que tienes la palabra a partir de ahora.

FEDRA: ¡Oh madre desdichada! ¿Qué deseo tuviste!

(Hip. vv. 332-337)

Estos ejemplos de plurales de modestia resultan especialmente interesantes para el propósito que se persigue en este trabajo, puesto que muestran una alternancia total en significado con el singular, es decir, podrían expresarse perfectamente en primera persona del singular, como se ve en el segundo de los textos, dado que el referente es el mismo en ambos casos; sin embargo, no son intercambiables desde el punto de vista pragmático. En (6a) se desprende la intención por parte del hablante de hacer al interlocutor partícipe y al mismo tiempo cómplice de los actos de aquel. 
Este plural de modestia, por tanto, evita el hecho de que el emisor se señale a sí mismo como responsable único de la acción y establece mayor distancia entre él mismo y la situación que describe. Su empleo resulta una estrategia pragmática que, como señala Haverkate 1994, p. 138, «representa una forma mitigadora de comportamiento verbal. Específicamente la finalidad primaria del hablante que se vale del recurso es evitar un enfrentamiento directo con el interlocutor».

Aunque quizá se podría plantear la duda de si no podrían ser plurales mayestáticos, el sentido general del pasaje nos da la clave y descarta totalmente esa idea. Los plurales mayestáticos se referirían a aquellos en los que el uso de la primera persona del plural se muestra como una marca simbólica de distinción -tradicionalmente era utilizado por individuos con un estatus social elevado-, mientras que en este caso se puede apreciar que el hablante no intenta mostrar superioridad frente a su interlocutor, sino evitar las responsabilidades que la acción requiere.

La idea de no responsabilidad por parte del hablante ante el hecho descrito encuentra relación directa con otro de los usos del plural, que se corresponde con un plural exclusivo, mediante el cual se hace referencia al hablante mismo y al conjunto de los mortales. Su actuación, en este caso, se ve respaldada por el hecho de que es mortal y que, por tanto, puede errar -en muchas situaciones sus acciones, además, son producto de la intervención de los dioses-.

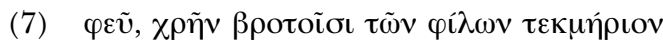

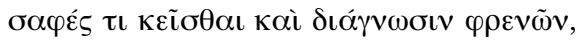

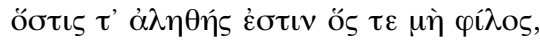

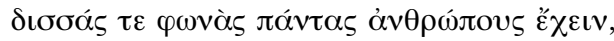

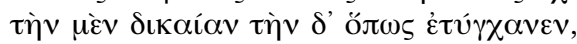

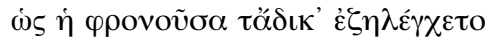

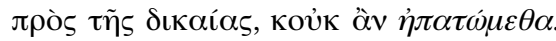

¡Ay! Menester sería que los mortales tuvieran un indicio claro respecto a los amigos y un conocimiento completo de sus intenciones: quién es verdadero amigo y quién no; y, además, que todos los hombres poseyeran dos voces distintas: una justa, y la otra de cualquier forma, para que por la justa fuera refutada la que alberga injustas intenciones. Pues así no resultaríamos engañados.

(Hip. vv. 925-931)

El uso de la primera persona del singular por parte de los personajes libres, además, aparece en dos clases de contextos. En primer lugar, se encuentra en situaciones comunicativas en las que impera el monólogo, o en contextos de diálogo en los que, ya porque el personaje es de rango inferior -que es lo que sucede normalmente-, ya porque se está poniendo en duda su identidad, el personaje en cuestión debe dejar

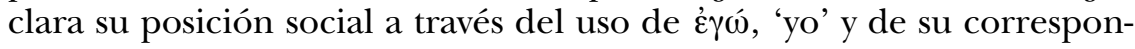


diente forma verbal. Es decir, la primera persona del singular viene dada, bien por un intento de marcación de poder sobre personajes de rango inferior, bien para destacar la identidad del emisor, como sucede, por ejemplo, en el agón que Hipólito mantiene con su padre, Teseo.

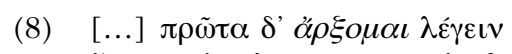

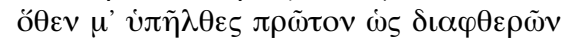

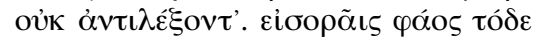



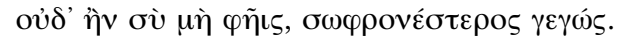

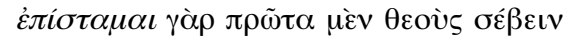

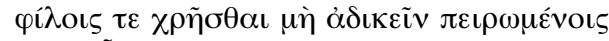

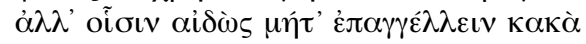

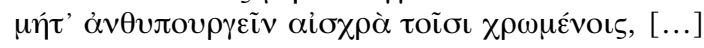

Empezaré a hablar por donde primero me atacaste en la idea de aniquilarme sin que yo te contestara. Contemplas esta luz y tierra: en ellas no ha nacido varón más prudente que yo, aunque tú afirmes que no. Efectivamente, sé, en primer lugar, venerar a los dioses, y, además, tener amigos que no tratan de violar la justicia, sino que sienten vergüenza de propalar infundios y de dar pago vil a quienes tratan. [...]

(Hip. vv. 991-999)

Es importante también destacar que entre los usos de la primera persona del singular por parte de los distintos personajes -especialmente los libres- en contextos de diálogo se han introducido aquellas intervenciones que reflejan una invocación o lamento dirigido a un dios. Estos textos, claro está, no se incluyen dentro de los parámetros habituales de estudio que se han manejado a lo largo de este trabajo en referencia a la imposición de un personaje sobre otro a través del uso del singular, sino que simplemente responden al uso tipo utilizado para tal fin, atendiendo a la comprensión final de la situación tras haber sido explicada por la divinidad que aparece como dea ex machina-ejemplo 9-.

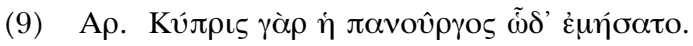

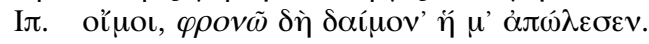

ÁrTemis: Porque la malvada Cipris así lo urdió.

Hipólito: ¡Ay de mí! Entiendo ya qué divinidad me ha aniquilado.

(Hip. vv. 1400-1401)

Una vez analizados los contextos en los que nos encontramos con el uso de la primera persona del singular y del plural por parte de los personajes libres, pasemos ahora al de los no libres. Hasta ahora, se ha visto que lo más destacable es que aquellos acuden en mayor medida al singular en detrimento del uso del plural, mientras que en los personajes no libres esta diferencia no resulta tan evidente. 
Es importante observar, pues, en qué situaciones acuden estos últimos al singular; dejando a un lado los ejemplos del Sirviente -tres, de atribución dudosa, puesto que algunas ediciones adjudican estos versos al Coro, y además, se encuentran en un contexto coral en el que la música y armonía probablemente fuesen más importantes que las propias palabras del personaje-, nos podemos concentrar en el uso del Mensajero en los contextos de diálogo. Este acude a la primera persona del singular para mostrar su sumisión ante su interlocutor, y es, una vez más, el contexto el que nos da cuenta de ello.

Los verbos que se localizan en primera persona del singular corresponden a preguntas directas al Coro para obtener información y verbos de existencia a través de los que da muestras de humildad, obediencia y respeto-ejemplo (10)-.

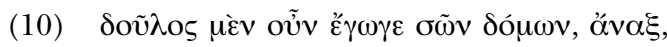

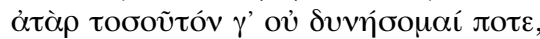

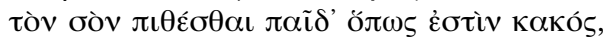

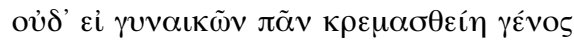

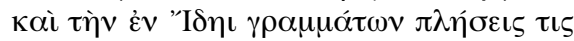

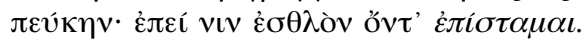

Yo, en verdad, soy un esclavo de tu mansión, señor, pero jamás podré creer que tu hijo es malvado, ni siquiera aunque se ahorcara toda la raza de las mujeres, ni aunque alguien llenara de notas la pineda del Ida, pues sé que es honrado.

(Hip. vv. 1249-1254)

El uso de la primera persona del singular por parte de la Nodriza, por otra parte, es un tanto complicado. La Nodriza es un personaje puente entre los libres y los no libres; aunque pertenece, de hecho, a estos últimos, la incapacidad de su señora y las características de su personaje ${ }^{31}$, hacen que en muchas ocasiones esta actúe asumiendo el papel de la protagonista, Fedra.

El hecho de que el empleo de dicha persona aparezca en los contextos de diálogo con su ama permite pensar que este se produce por dos causas fundamentales: por un lado, intensificar su identidad para llevar las riendas de la situación -lo que también aparece en contextos de monólogo-, y por otro, infundir el grado de confianza suficiente que le falta a Fedra. Esta idea cobra fuerza al observar que, además, en la mayoría de las ocasiones, se trata de verbos de acción que afectan directamente a aquella, aunque en muchos casos estos muestren resig-

\footnotetext{
31 El ser un personaje mayor hace que la tensión sexual que aparece como motor principal de la obra en algunos personajes no se manifieste en ella, lo cual le otorga también un estatus superior al de otros personajes.
} 
nación e incapacidad de resolución por parte de la esclava ante la gravedad de la situación por la que atraviesa Fedra ${ }^{32}$.



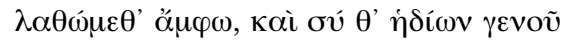

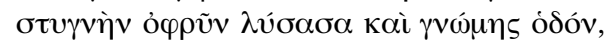

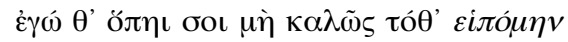

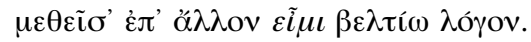

¡Vamos, querida hija, olvidémonos ambas de nuestras anteriores palabras y sé tú más agradable, eliminando tu ceño hostil y la resolución de tu mente; y yo, dejando la ruta por donde te he seguido hasta ahora sin acierto, acudiré a un lenguaje mejor!

(Hip. vv. 288-292)

El último personaje que queda por analizar de los que se pueden considerar dentro del grupo de los no libres es el Coro. A pesar de introducirse dentro de una categoría social más baja, cabe tener en cuenta que se trata de un personaje tipo que responde a unas reglas de intervención y expresión muy específicas.

Aunque podría esperarse que el hecho de ser un personaje plural llevaría a que la persona verbal que escogiese para expresarse fuese la primera del plural y que quizá pudiese aparecer la primera persona del singular en los pasajes que fuesen pronunciados por el Corifeo, cabe recordar que en Tragedia esta no es la norma habitual, y los datos que aquí se plantean no hacen sino confirmar esta tendencia.

Las intervenciones del Corifeo en Hipólito vienen marcadas por el uso de dicha forma en plural, excepto en el verso 540, en el que es el Coro el que acude a él; no obstante, los miembros del Coro no se refieren a ellos mismos como entidad plural en este ejemplo, sino al conjunto de todos los mortales, como se puede observar.

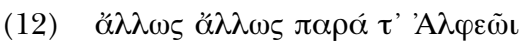

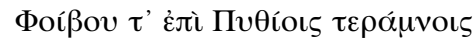

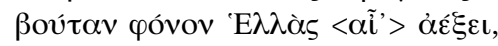

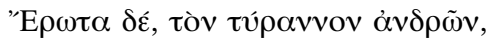

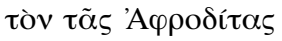

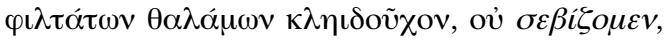

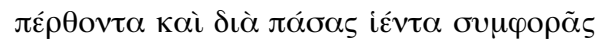

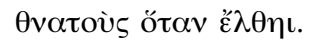

En balde, en balde, sí, junto al Alfeo y en la mansión pítica de Febo la Hélade incrementa el sacrificio de toros; en cambio, a Eros, el ti-

\footnotetext{
${ }^{32}$ El amor que Fedra siente por su hijastro Hipólito le provoca tal dolor que se ve inmersa en una profunda enfermedad.
} 
rano de los humanos, el que posee las llaves del gratísimo tálamo de Afrodita, no lo veneramos, aunque es devastador y acarrea todo tipo de desgracias a los mortales, cuando llega.

(Hip. vv. 535-542)

Por otra parte, el hecho de que el Corifeo utilice el plural resulta lógico, puesto que a través de este uso se pretende enfatizar su papel como representante de un elemento conjunto dentro de la tragedia. Dicho empleo del plural, además, se hace patente en las primeras intervenciones del personaje con la intención de dejar clara su identidad ante el público para que este lo reconozca dentro de su rol de ahí en adelante.

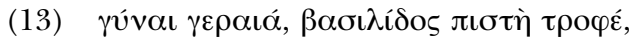

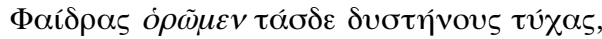

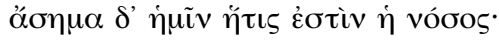

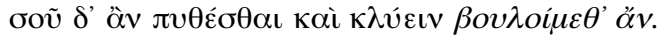

Anciana mujer, fiel nodriza de la reina Fedra, observamos la desdichada situación, pero nos resulta incierto cuál es su afección. Quisiéramos enterarnos y oírlo de ti.

(Hip. vv. 267-270)

El uso de la primera persona del singular por parte del Coro se ve justificado por varias razones; en primer lugar, la intención de este de que su intervención como personaje plural sea recibida por el público como unidad. En segundo lugar, podría vincularse a la relación entre el singular y el poder. La máxima que versa la información es poder puede asociarse en este punto con el uso del singular por parte del Coro; la posesión de información por parte de este le permite acudir a esta primera persona del singular para expresarse.

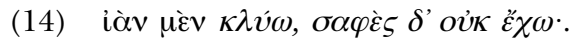

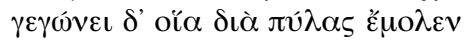

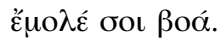

Oigo gritos, pero no los comprendo bien. Mas está claro por dónde te han venido sus voces. Por las puertas te han venido.

(Hip. vv. 585-587)

Asimismo, parece que la semántica del verbo influye en la elección del singular y del plural, de manera que el Coro y el Corifeo acuden con mayor frecuencia al singular en su utilización de términos que se refieren a las emociones del personaje, aquellos que tienen que ver con percepción sensorial, y los que se relacionan con el pensamiento reflexivo, tal y como nota Kaimio 1970, y se puede ver en el ejemplo anterior, así como en el que sigue. 


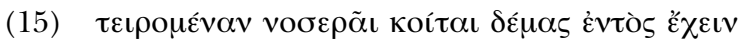

oǔk $\omega v, \lambda \varepsilon \pi \tau \grave{\alpha} \delta \dot{\varepsilon} \varphi \alpha ́ \rho \eta$

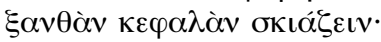

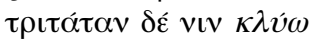

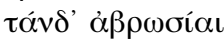

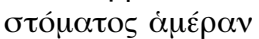

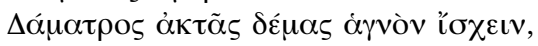

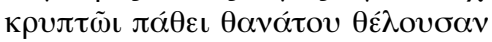

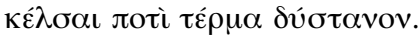

Que afligida de enfermiza postración retiene su cuerpo dentro de palacio, y ligeros velos ocultan su rubia cabeza. Oigo decir que desde hace tres días, sin alimentar su boca, mantiene su cuerpo ayuno del fruto de Deméter, deseando arribar, a causa de oculto sufrimiento, al desdichado fin de la muerte.

(Hip. vv. 131-140)

No obstante, el propio Kaimio admite que se trata de un estudio delicado en el que las pautas que parecen presentarse no resultan demasiado precisas en lo que se refiere a la elección de las formas de primera persona del singular y del plural.

\section{ConClusión}

En primer lugar, es preciso recordar que las comparaciones entre el uso de la primera persona del singular y la primera persona del plural entre los distintos personajes de Hipólito deben ser consideradas solo en aquellos contextos en los que existe un diálogo explícito entre ellos, de manera que se puedan comparar los usos de unos y otros en relación con su poder social y el de su interlocutor.

En general, se puede afirmar que existen pequeñas diferencias en la utilización de estas personas verbales dependiendo del nivel de importancia social del personaje en cuestión, así como de la semántica de los verbos que se utilicen, que se hace patente especialmente en las intervenciones del Coro y el Corifeo.

La primera persona del singular encuentra una mayor frecuencia de empleo en los personajes libres que en los no libres -como muestra de identidad y poder-, mientras que en estos últimos el uso del singular es menos numeroso proporcionalmente en comparación con el plural.

En cuanto al parámetro de género, se observa que el uso del plural en los personajes libres femeninos está caracterizado por el llamado «plural de modestia». Además, dado que los ejemplos de este uso se localizan en contextos de diálogo entre personajes femeninos, Fedra, la Nodriza y el Coro, se podría afirmar que las diferencias sociales entre las mujeres no se muestran de forma tan clara como las que se pro- 
ducen entre los hombres. Tal conclusión se extrae del hecho de que los números que se presentan en el empleo del singular y aquellos que se refieren al plural difieren más en las intervenciones de los personajes masculinos -tanto libres como no libres- que en las de los personajes femeninos.

Asimismo, cabe destacar que en los personajes no libres no existe una diferencia entre el uso del singular y del plural tan evidente como la que se encontraba en los personajes libres, al mismo tiempo que, en el uso que aquellos hacen del singular, su utilización viene condicionada por la manifestación evidente de su condición como muestra de obediencia y sumisión.

Siguiendo la línea que se ha propuesto hasta el momento, cabe concluir que los parámetros sociolingüísticos, especialmente en lo que se refiere al de jerarquía social, tienen cierta influencia en la elección del uso de la primera persona del singular y del plural, siempre teniendo muy en cuenta el contexto en el que se utilizan, la situación social del interlocutor al que se dirigen y la semántica del verbo. El tratamiento de los números expuestos en las tablas de forma independiente, esto es, sin un estudio específico de los contextos, puede dar lugar a falsas conclusiones que se disipan al acudir directamente a las fuentes.

\section{REFERENCIAS BIBLIOGRÁFICAS}

Benveniste, É. (2004, 1971): Problemas de lingüistica general, Mexico D. F., Siglo XXI editores.

Berman, L. (1998): Speaking through the silence. Narratives, social conventions and power in Java, Oxford, Oxford University Press.

CALAme, C. (1995): «Dalla poesia coralle allo stasimo tragico: funzione pragmatica di voci femminili» en Martino, F. de y Sommerstein, A. H. (ed.), Lo spettacolo delle voci, Bari, Levante Editori, Vol. I, pp. 79-101.

Crespo, E. (coord.) (2004): Esquilo, Sófocles, Eurípides. Obras Completas, Madrid, Cátedra.

Dysouw, M. (2003): The paradigmatic structure of person marking, Oxford, Oxford University Press.

Diggle, J. (ed.) (1984): Euripidis Fabulae. Cyclops, Alcestis, Medea, Heraclidae, Hippolytus, Andromacha, Hecuba. Tomus I, Oxford, Oxford University Press.

Gildersleeve, B. L. (1900): Sintax of Classical Greek. From Homer to Demosthenes, Part I. Nueva York, Cincinnati, Chicago, American Book Company.

GILI Y GAYA, S. (1961): Curso superior de sintaxis española, Barcelona, Spes.

Greimas, A. J. y Courtés, J. (1990, 1979): Semiótica. Diccionario razonado de la teoría del lenguaje, Madrid, Gredos.

Haverkate, H. (1994): La cortesía verbal. Estudio pragmalingüístico, Madrid, Gredos.

KAIMIO, M. (1970): The chorus of Greek drama within the light of the person and number used, Helsinki-Helsingfors, Societas Scientiarum Fennica. 
LYONS, J. (1968): Introduction to theoretical linguistics, Cambridge, Cambridge University Press.

Lasso de la Vega, J. S. (1968, 1967): Sintaxis griega, Madrid, CSIC, Patronato Menéndez y Pelayo.

MCCLuRE, L. (1995): «Female speech and characterization in Euripides» en de Martino, F. y Sommerstein, A. H. (eds.), Lo spettacolo delle voci, Vol. II, Bari, Levante Editori, pp. 35-60.

- (1999): Spoke like a woman: Speech and gender in Athenian drama, Nueva Jersey, Princeton.

Minchin, E. (2007): Homeric voices. Discourse, memory, gender, Oxford, Oxford University Press.

SCHIfFrin, D. (1987): Discourse markers, Cambridge, Cambridge University Press. 
